\title{
Malignant gastrointestinal neuroectodermal tumor with BRAF mutation and a history of malignant melanoma: A case report
}

\author{
TOSHINARI YAGI $^{1}$, SHIGENORI NAGATA $^{2}$, TAKASHI YAMAMOTO ${ }^{3}$, \\ TORU WAKAMATSU ${ }^{4}$, YOSHINORI IMURA ${ }^{4}$, HIRONARI TAMIYA ${ }^{4}$, \\ HIDEAKI SABE ${ }^{4}$, KATSUNARI YAMASHITA ${ }^{4}$ and SATOSHI TAKENAKA ${ }^{4}$ \\ Departments of ${ }^{1}$ Outpatient Chemotherapy, ${ }^{2}$ Diagnostic Pathology and Cytology, ${ }^{3}$ Clinical Laboratory, \\ and ${ }^{4}$ Orthopedic Surgery, Osaka International Cancer Institute, Osaka 541-8567, Japan
}

Received May 30, 2020; Accepted October 21, 2020

DOI: $10.3892 / \operatorname{mco} .2020 .2185$

\begin{abstract}
Malignant gastrointestinal neuroectodermal tumors (GNETs), also called clear-cell sarcoma-like tumors of the gastrointestinal tract, are rare and highly aggressive tumors originating in the gastrointestinal tract. These tumors are generally immunohistochemically positive for S-100 protein (S-100) and SRY-related HMG-box 10 (SOX10), and often contain EWSR1-ATF1 or EWSR1-CREB1. The histological features of GNETs overlap with those of clear-cell sarcoma of the tendons and aponeuroses. However, GNETs immunohistochemically lack melanocyte-specific markers and often demonstrate positivity for CD56, synaptophysin and neuron-specific enolase. The present case reports a woman with a history of desmoplastic malignant melanoma exhibiting a BRAF mutation, which later transformed into a GNET of the small intestine with both a BRAF mutation and two subtypes of EWSR1-ATF1 fusion genes. Tumor cells were revealed to
\end{abstract}

Correspondence to: Dr Toshinari Yagi, Department of Outpatient Chemotherapy, Osaka International Cancer Institute, 3-1-69 Otemae, Chuo-ku, Osaka 541-8567, Japan

E-mail: yagi-to@mc.pref.osaka.jp

Abbreviations: GNET, malignant gastrointestinal neuroectodermal tumor; CCSLGT, clear-cell sarcoma-like tumor of the gastrointestinal tract; CCS, clear-cell sarcoma; MM, malignant melanoma; NR, normal range; RT-PCR, reverse transcription polymerase chain reaction; $\mathrm{H} \& \mathrm{E}$, hematoxylin and eosin; HMB45, human melanin black-45; N/C, nuclear/cytoplasm; S-100, S-100 protein; SOX10, SRY-related HMG-box 10; cKIT, stem cell factor receptor; CK, cytokeratin; $\mathrm{CD}$, cluster of differentiation; $\alpha$-SMA, $\alpha$-smooth muscle actin; DOG1, discovered on GIST1; TTF-1, thyroid transcription factor-1; GCDFP-15, gross cystic disease fluid protein-15; EMA, epithelial membrane antigen; MyoD1, myogenic differentiation 1; PAX8, paired box 8 ; ER, estrogen receptor; IHC, immunohistochemistry

Key words: malignant gastrointestinal neuroectodermal tumor, clear cell sarcoma-like tumor of the gastrointestinal tract, malignant melanoma, BRAF, EWSR1-ATF1, dabrafenib mesylate, trametinib dimethyl sulfoxide be weakly immunoreactive or negative for S-100 and SOX10, lacked markers of melanocytic differentiation and were focally positive for CD56. Combination therapy with dabrafenib mesylate and trametinib dimethyl sulfoxide proved to be temporarily effective against this tumor. The present case is relatively unique as, to the best of our knowledge, there is no case of GNET with a history of melanoma. Furthermore, there is no report of GNET exhibiting both a BRAF mutation and an EWSR1-ATF1 fusion gene. Further accumulation of similar cases is necessary to elucidate the pathological significance of this GNET having a BRAF mutation.

\section{Introduction}

Malignant gastrointestinal neuroectodermal tumor (GNET) is a term designated by Stockman et al for rare, clear-cell sarcoma-like tumors of the gastrointestinal tract (CCSLGT) (1). They reappraised CCSLGT cases and clarified the following features. This tumor is histologically characterized by a sheet-like or nested population of epithelioid or oval-to-spindle-shaped cells with small nucleoli and scattered mitoses. These tumor cells are positive for S-100 protein (S-100), SRY-related HMG-box 10 (SOX10), and vimentin and sometimes positive for CD56, synaptophysin, neuroblastoma 84, neuron-specific enolase (NSE), and neurofilament proteins. They usually contain EWSR1 gene rearrangements, such as EWSR1-ATF1 or EWSR1-CREB1. In general, GNET lacks melanocyte-specific markers, making it clearly different from clear-cell sarcoma (CCS) of the tendons and aponeuroses.

Because GNETs are extremely rare and reports on them are only available in the form of case reports or small size reviews, their morbidity is unclear. They tend to occur mainly in young to middle-aged adults. The most common site of tumor origin is the small intestine (57.9\%), followed by the stomach, colon, and other sites of the gastrointestinal tract (2). Surgery is often the choice for resectable lesions, but there are currently no standard chemotherapeutic or targeted therapeutic options for this disease in the metastatic setting. The prognosis is generally poor, and the median survival was reported to be 9.5 months (3). Here, we present a unique case of a GNET that has a history of desmoplastic malignant melanoma exhibiting 
a BRAF mutation, which later transformed into a GNET of the small intestine with both a BRAF mutation and two subtypes of the EWSR1-ATF1 fusion gene.

\section{Case report}

In April 2018, a 66-year-old woman with multiple metastatic tumors was referred to our hospital for further diagnosis and treatment. She had suffered from lower abdominal pain in February 2018 and consulted a nearby hospital. Imaging examinations revealed a tumor in the small intestine, a soft-tissue mass in her left forearm, a bilateral pleural mass, and a left breast mass. A small intestinal tumor of $8 \mathrm{~cm}$ in diameter was resected in early March 2018, and the histological diagnosis was undifferentiated carcinoma. She was diagnosed with carcinoma of unknown primary and transferred to our hospital.

The patient also suffered from idiopathic thrombocytopenic purpura and had a history of cutaneous malignant melanoma (MM). Her MM history was as follows: She underwent extended resection of $\mathrm{MM}$ at her left thigh in April 2010 at another hospital; she also received adjuvant combination chemotherapy containing dacarbazine, nimustine hydrochloride, vincristine and interferon- $\beta$. However, the patient experienced a local recurrence of MM in May 2011 and underwent additional extended resection, though no adjuvant chemotherapy was administered after the second operation.

The patient's pleural mass was rapidly growing; hence, she was admitted to our hospital in April 2018. On admission, she was slightly obese (the body mass index was 28.06). Physical examination demonstrated a soft, painful mass of $5 \mathrm{~cm}$ in diameter in the flexor muscle side of her left forearm. In addition, a hard mass of $2 \mathrm{~cm}$ in diameter was palpable in the left upper portion of her left breast. Computed tomography revealed a large necrotic tumor of $15 \mathrm{~cm}$ in diameter in the left upper portion of her chest, with bilateral multiple lung metastatic masses and left pleural effusion. Furthermore, a 47-mm tumor without contrast effect in the left forearm and a 17-mm nodule in the left breast were detected, but no lymphadenopathy was found.

Laboratory studies yielded the following results: White blood cells $=7.96 \times 10^{9} / 1$, hemoglobin $=12.8 \mathrm{~g} / \mathrm{dl}$, platelet count $=79 \times 10^{9} / 1$ (normal range [NR]: $\left.158-348 \times 10^{9} / 1\right)$, lactate dehydrogenase=438 IU/1 (NR: 124-222 IU/1), C-reactive protein=6.03 mg/dl $(\mathrm{NR} \leq 0.14 \mathrm{mg} / \mathrm{dl}), \mathrm{CA}-125=38 \mathrm{U} / \mathrm{ml}(\mathrm{NR}$ $\leq 35 \mathrm{U} / \mathrm{ml}), \mathrm{NSE}=19.4 \mathrm{ng} / \mathrm{ml}(\mathrm{NR} \leq 16.3 \mathrm{ng} / \mathrm{ml})$, and platelet-associated $\mathrm{IgG}=59 \mathrm{ng} / 10^{7}$ cells ( $\mathrm{NR} \leq 46 \mathrm{ng} / 10^{7}$ cells).

Reexamination of the resected small intestinal tumor by our hospital pathologists resulted in the new diagnosis of a GNET (details of the findings are described in the next section), as reverse-transcription polymerase chain reaction (RT-PCR) of the specimen revealed an EWSR1 exon8/ATF1 exon4 fusion transcript, which is disease-specific for GNET or CCS. Additional biopsies of her left forearm mass and left breast mass were performed at our hospital. The histopathological diagnosis of the left forearm mass was the same as that of the tumor of the small intestine. On the other hand, the breast mass was diagnosed as invasive ductal carcinoma (papillotubular carcinoma).
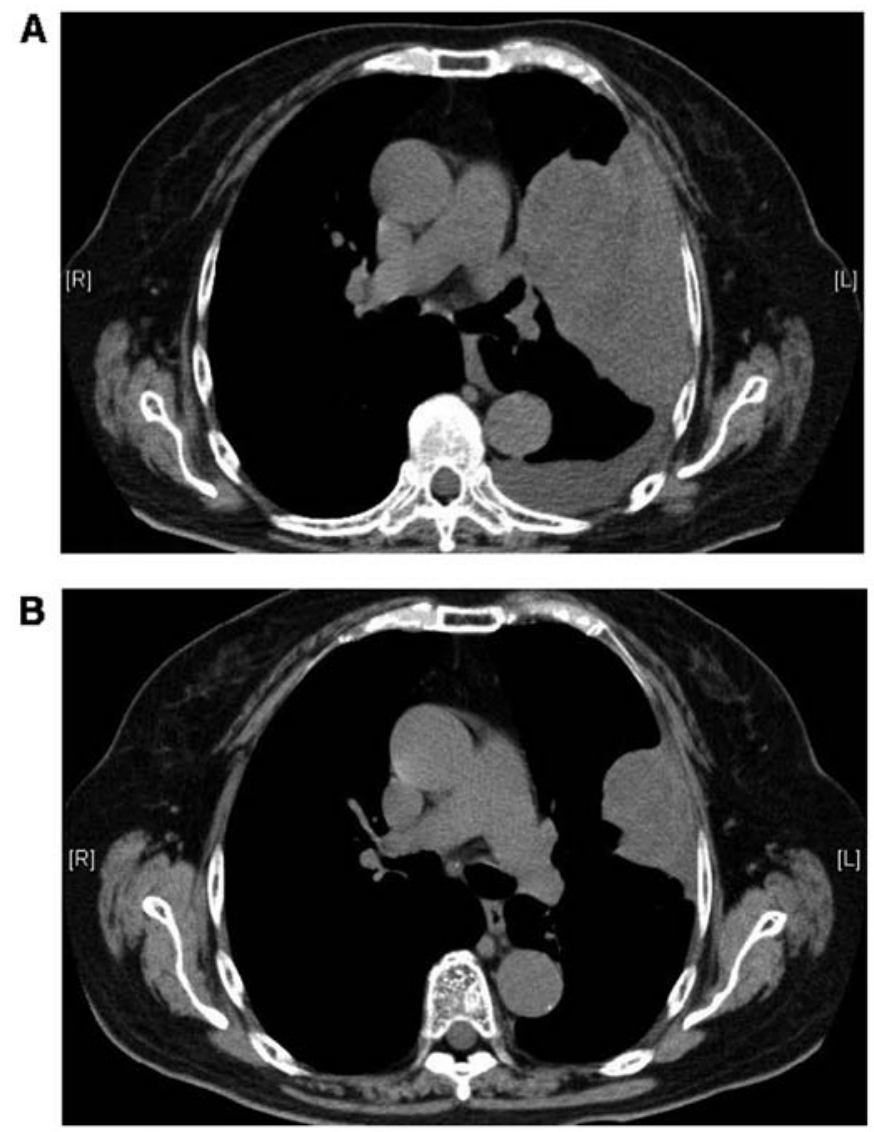

Figure 1. CT scan before and after treatment with dabrafenib mesylate and trametinib dimethyl sulfoxide. (A) CT scan before the treatment, (B) CT scan taken 3 months after starting the treatment.

At this point, we questioned whether the previous diagnosis of MM was valid because it showed phenotypic and immunohistochemical overlap with GNET and CCS. Therefore, we asked the hospital which had treated her MM to provide samples. Because of the rapid progression of her pleural mass, chemotherapy with doxorubicin was started even before the samples were examined. Two courses of doxorubicin were administered, stabilizing the case. However, the patient further suffered from pneumocystis pneumonia, and it was important to discontinue chemotherapy. She was further treated using a drug combination of sulfamethoxazole and trimethoprim and recovered from pneumonia.

In June 2018, the histological diagnosis of the previous samples at the time of recurrence of MM was finally established as desmoplastic MM with a BRAF mutation. Therefore, the BRAF gene status of the small intestinal and left forearm tumors were checked, and both tissues were found to exhibit a BRAF mutation. Her GNET was diagnosed as double-positive for BRAF mutation and EWSR1-ATF1 fusion transcript. Dabrafenib mesylate and trametinib dimethyl sulfoxide were started, a combination therapy that proved effective and achieved a partial response three months later (Fig. 1). The size of the pleural mass increased in January 2019, and these drugs were replaced by immunotherapy with nivolumab and ipilimumab. This immunotherapy was performed only once because of the rapid progression of her pleural mass, the occurrence of multiple brain metastases, and the deterioration of her health. 
Table I. Summary of immunohistochemical and genetic findings.

\begin{tabular}{|c|c|c|c|c|}
\hline Variables & Sample 1 (Fig. 2) & Sample 2 & Sample 3 (Fig. 3) & Sample 4 (Fig. 4) \\
\hline Resection date & April 2010 & July 2011 & March 2018 & April 2018 \\
\hline Site & $\begin{array}{l}\text { Left thigh } \\
\text { (primary lesion) }\end{array}$ & $\begin{array}{l}\text { Operation scar in the } \\
\text { left thigh (recurrent site) }\end{array}$ & Small intestine & $\begin{array}{l}\text { Soft-tissue mass in the } \\
\text { left forearm }\end{array}$ \\
\hline Pathological diagnosis & $\begin{array}{l}\text { Desmoplastic } \\
\text { MM }\end{array}$ & $\begin{array}{l}\text { Recurrence of } \\
\text { desmoplastic MM }\end{array}$ & GNET & $\begin{array}{l}\text { Metastatic lesion of } \\
\text { GNET }\end{array}$ \\
\hline IHC stain: Positive & S-100 & $\begin{array}{l}\text { S-100, SOX10, Melan A } \\
(+, \text { focal })\end{array}$ & $\begin{array}{l}\text { S-100 (-/+, a few), SOX10 } \\
(+, \text { scattered), CK AE1/3 } \\
(+, \text { focal }), \text { CK CAM5.2 } \\
(+, \text { focal }), \text { CD56 (+, focal })\end{array}$ & CD56 (+, focal $)$ \\
\hline IHC stain: Negative & HMB45 & HMB45, cKIT & $\begin{array}{l}\text { CK7, CK20, melan A, } \\
\text { HMB45, desmin, } \alpha-S M A, \\
\text { HHF35, caldesmon, cKIT, } \\
\text { DOG1, CD34, TTF-1, } \\
\text { GCDFP-15, EMA, MyoD1, } \\
\text { myogenin, synaptophysin, } \\
\text { chromogranin A, PAX8, ER }\end{array}$ & $\begin{array}{l}\text { S-100,SOX10, HMB45, } \\
\text { Melan A }\end{array}$ \\
\hline Ki-67 labeling index & No sample & $5 \%$ & $50 \%$ & Not performed \\
\hline $\begin{array}{l}\text { Genetic findings } \\
\text { of BRAF }\end{array}$ & No sample & $\begin{array}{l}\text { BRAF mutation } \\
\text { (V600E) }\end{array}$ & BRAF mutation (V600E) & $\begin{array}{l}\text { BRAF mutation } \\
(\mathrm{V} 600 \mathrm{E})\end{array}$ \\
\hline $\begin{array}{l}\text { Genetic findings of } \\
\text { EWSR 1/ATF1 }\end{array}$ & No sample & - & EWSR1 exon8/ATF1 exon4 & $\begin{array}{l}\text { EWSR } 1 \text { exon10/ATF1 } \\
\text { exon5 }\end{array}$ \\
\hline
\end{tabular}

MM, malignant melanoma; GNET, malignant gastrointestinal neuroectodermal tumor; IHC, immunohistochemistry; S-100, S-100 protein; SOX10, SRY-related HMG-box 10; cKIT, stem cell factor receptor; CK, cytokeratin; $\alpha$-SMA, $\alpha$-smooth muscle actin; DOG1, discovered on GIST1; TTF-1, thyroid transcription factor-1; GCDFP-15, gross cystic disease fluid protein-15; EMA, epithelial membrane antigen; MyoD1, myogenic differentiation 1; PAX8, paired box 8; ER, estrogen receptor. HMB45, human melanin black-45; HHF35, muscle actin (clone: HHF35); CAM 5.2, low molecular weight cytokeratin (clone: CAM 5.2); EWSR1, Ewing sarcoma breakpoint region 1; ATF1, activating transcription factor 1; BRAF, v-raf murine sarcoma viral oncogene homolog B1.

She was transferred to a hospice facility in February 2019 and died in January 2020 because of the deterioration of her brain metastasis.

\section{Pathological findings}

In total, four tissue samples were reviewed (Table I): A primary cutaneous lesion of the left thigh resected in April 2010 (Sample 1), a recurrent tumor embedded in the former operation scar excised in July 2011 (Sample 2), a small intestinal mass resected in March 2018 (Sample 3) and a core-needle biopsy of a soft-tissue mass that arose in the left forearm in April 2018 (Sample 4).

i) Primary cutaneous lesion of the left thigh (Sample 1). Sections stained with hematoxylin and eosin (H\&E) showed a subpedunculated polypoid lesion with a maximum diameter of $6 \mathrm{~mm}$ associated with intraepidermal neoplasm harboring brown pigments at the tumor border (Fig. 2A). The protrusion was mainly composed of spindle cells with relatively low atypia proliferating in fascicles (Fig. 2B). No lymphovascular invasion was identified. Immunohistochemically, the tumor cells were positive for $\mathrm{S}-100$ but negative for human melanin black-45 (HMB45) (Fig. 2C and D). The histological diagnosis was desmoplastic MM with an unclear surgical margin. No tissue blocks were available for additional studies.

ii) Recurrent tumor beneath operation scar in the left thigh (Sample 2). Sections stained with H\&E showed the proliferation of atypical spindle cells in the subcutaneous tissue. The morphological features resembled that of the invasive component of the previous tumor (Sample 1). Infiltrating cells were immunoreactive to S-100 and SOX10. Melan A was focally positive, although HMB45 was consistently negative. The Ki-67 labeling index was 5\%. These features are compatible with the local recurrence of desmoplastic MM.

iii) Small intestinal tumor (Sample 3). Sections stained with $\mathrm{H} \& \mathrm{E}$ demonstrated a well-demarcated tumor localized in the intestinal subserosa, which was composed of small round cells with a high nuclear/cytoplasm (N/C) ratio and frequent mitoses accompanied by central necrosis, focal hemorrhage, and myxoid changes (Fig. 3A). Neoplastic cells showed an immunophenotype with limited expressions of S-100 and SOX10 and negativity for HMB45 and Melan A (Fig. 3B-E). Other immunohistochemical findings were as follows: CK AE1/3(+, focal), CK CAM5.2(+, 

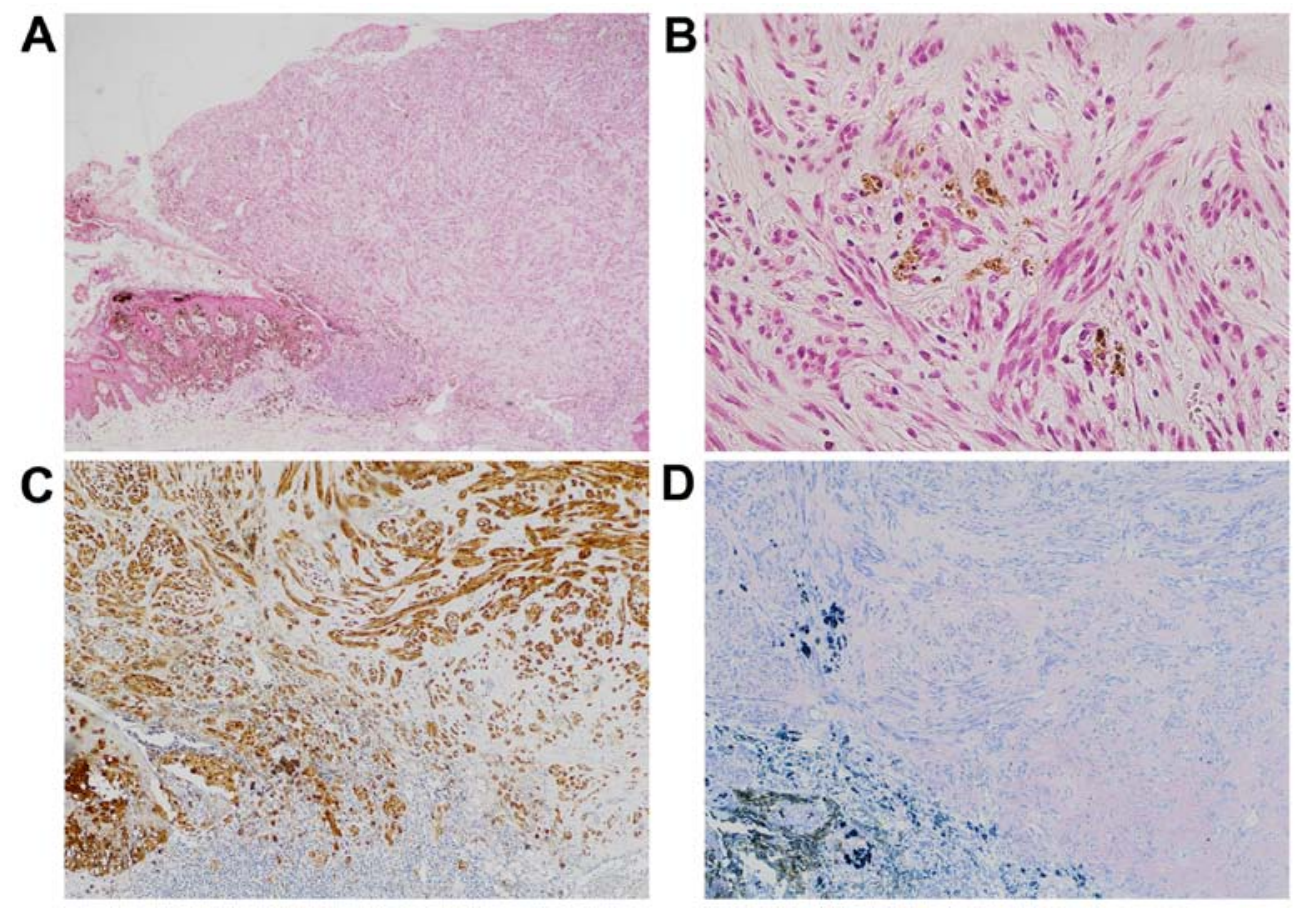

Figure 2. Photomicrographs of the desmoplastic melanoma of the left thigh. (A) Subpedunculated polyp associated with intraepidermal neoplasm harboring brown pigments at the border (H\&E; magnification, x20). (B) The mass was mainly composed of spindle cells with blunt nuclear atypia proliferating in fascicles mixed with a fibrocollagenous matrix (H\&E; magnification, x200). The mass was immunohistochemically positive for (C) S-100 protein (IHC stain; magnification, x40) but negative for (D) HMB45 (IHC stain; magnification, x40). HMB45, human melanin black-45; IHC, immunohistochemical.

focal), CD56(+, focal; Fig. 3F), CK7(-), CK20(-), Desmin(-), $\alpha-\operatorname{SMA}(-), \operatorname{HHF} 35(-)$, Caldesmon(-), cKIT(-), DOG1(-), CD34(-), TTF-1(-), GCDFP-15(-), EMA(-), MyoD1(-), Myogenin(-), Synaptophysin(-), Chromogranin A(-), PAX8(-) and ER(-). The Ki-67 labeling index was 50\%. These features were suggestive of GNET, although the diagnosis was inconclusive.

iv) Soft-tissue mass of the left forearm (Sample 4). Sections stained with $\mathrm{H} \& \mathrm{E}$ revealed morphological features resembling those of Sample 3, composed of compact dysplastic cells with a high N/C ratio, which were immunohistochemically negative for S-100, SOX10, HMB45 and Melan A but focally positive for CD56 (Fig. 4A-F). A metastatic tumor was considered based on molecular rearrangement similar to that seen in the small-intestinal tumor (Sample 3), as described below.

\section{Genetic findings}

All BRAF mutation analyses were consigned to LSI Medience Corporation (Tokyo) and were performed using real-time PCR techniques. BRAF mutations (V600E) were detected in Samples 2, 3 and 4. To detect the type of EWSR1-ATF1 fusion transcript, RT-PCR was performed at our laboratory, as previously reported (4). In Sample 2, no fusion transcript was detected. However, in Sample 3, an EWSR1 exon8/ATF1 exon4 fusion transcript was identified. In addition, RT-PCR of Sample 4 revealed an EWSR1 exon10/ATF1 exon5 fusion transcript.

\section{Discussion}

Clear-cell sarcoma is a rare soft-tissue sarcoma that was first described by Enzinger (5). It typically involves the deep soft tissues of the extremities in close proximity with tendons and aponeurotic structures. Its distinctive features include a nested growth pattern and consistent melanocytic differentiation (6). From a pathological point of view, CCS and MM share many histological and immunohistochemical features. However, CCS and MM are currently considered to be two distinct disease entities because, in most cases, CCS involves specific fusion genes such as EWSR1-ATF1 or EWSR-CREB1. On the other hand, CCSLGT is an extremely rare condition that was originally described by Zambrano et al in 2003 as an osteoclast-rich tumor of the gastrointestinal tract with features resembling those of CCS of soft parts (7). Stockman et al (1) described the clinicopathological, immunohistochemical, ultrastructural and molecular analysis of 16 cases with CCSLGT. They reported that these tumors are positive for S-100 and SOX10 but lack melanocyte-specific markers. Genetically, these tumors were characterized by EWSR1 gene rearrangements, including EWSR1-ATF1 or EWSR1-CREB1 fusion, similar to CCS of the tendons and aponeuroses. At the ultrastructural level, they lacked evidence of melanocytic differentiation and showed features of neural differentiation. In their study, Stockman et al suggested designating these tumors as 'malignant gastrointestinal neuroectodermal tumors' (GNETs).

Our case exhibited the following unique characteristics: i) the patient had a history of BRAF-mutated desmoplastic $\mathrm{MM}$; ii) her tumor cells were weakly immunoreactive or negative for S-100 and SOX10; iii) there was no evidence of melanocytic differentiation; iv) focal positivity for CD56 was present; and v) her tumor exhibited both a BRAF mutation and two subtypes of EWSR1-ATF1. Thway and Fisher (8) reviewed the clinicopathological and molecular features of 

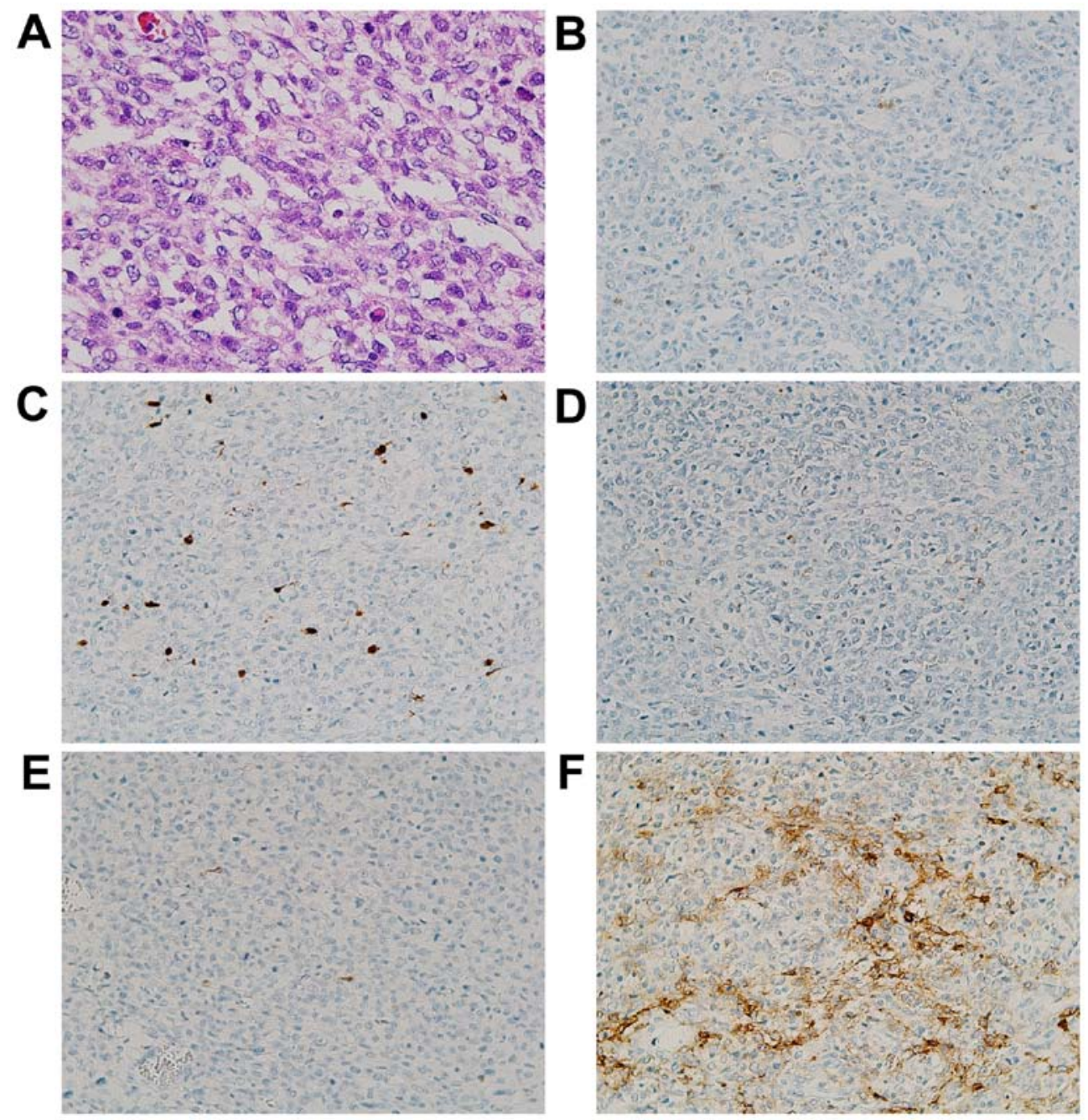

Figure 3. Photomicrographs of the small intestinal tumor. (A) A patternless proliferation of atypical cells characterized by scant pale cytoplasm with microvesicular degeneration (H\&E stain; magnification, x200). The tumor was immunohistochemically partially positive for (B) S-100 (IHC stain; magnification, x100) and (C) SOX10 (IHC stain; magnification, x100), but negative for (D) HMB45 (IHC stain; magnification, x100) and (E) Melan A (IHC stain; magnification, x100). Additionally, the tumor was positive for (F) CD56 (IHC stain; magnification, x100). IHC, immunohistochemical; SOX10, SRY-related HMG-box 10; HMB45, human melanin black-45.

five diverse neoplasms that frequently exhibit EWSR1-CREB1 or EWSR1-ATF1 genetic fusion: Angiomatoid fibrous histiocytoma, conventional CCS (of tendons and aponeuroses), CCSLGT, hyalinizing CCS of the salivary gland and primary pulmonary myxoid sarcoma. Among these five neoplasms, S-100 negativity was a finding consistent with angiomatoid fibrous histiocytoma and hyalinizing CCS of the salivary gland, but their pathological and clinical features were clearly different from those of our case. Clinicopathologically, our case was more likely to be diagnosed with CCS or GNET, but both neoplasms were reported to be positive for S-100 without exception. Therefore, we could not reach a definite diagnosis for our case. However, considering the tumor site (small intestine), the absence of melanocytic differentiation, and the weak positivity for CD56, we determined that her tumor was very similar to a GNET.

There were some limitations in our diagnosis. First, the patient already had three large tumors when she visited the previous hospital for the first time: A pleural mass, a small intestinal mass, and a soft-tissue mass in her left forearm. No biopsy of her pleural mass was performed. The EWSR1-ATF1 fusion gene subtypes were different between the small intestinal mass and the forearm mass. As for CCS, several cases with two or three different types of EWS-ATF1 fusion have previously been reported $(9,10)$. To the best of our knowledge, no case report of a GNET with more than two subtypes of EWSR1-ATF1 fusion exists so far. Therefore, it cannot be verified whether her tumor is really a single tumor or a collection of similar tumors. In addition, this tumor exhibited both a BRAF mutation and EWSR1-ATF1 fusion. Such cases have rarely been reported with CCS $(11,12)$; however, to the best of our knowledge, no case report of a GNET with both a BRAF mutation and EWSR1-ATF1 fusion gene exists so far. This tumor is similar to GNET, but it may be a different type of neoplasm.

The unique highlight in our case is the patient's history of desmoplastic MM. It should be noted that the patient's past MM and GNET are morphologically and immunohistochemically different neoplasms, but both tumors exhibited BRAF mutations. Therefore, our case suggests one hypothesis for the pathogenetic process of her GNET: Her desmoplastic MM exhibiting a BRAF mutation acquired an additional 


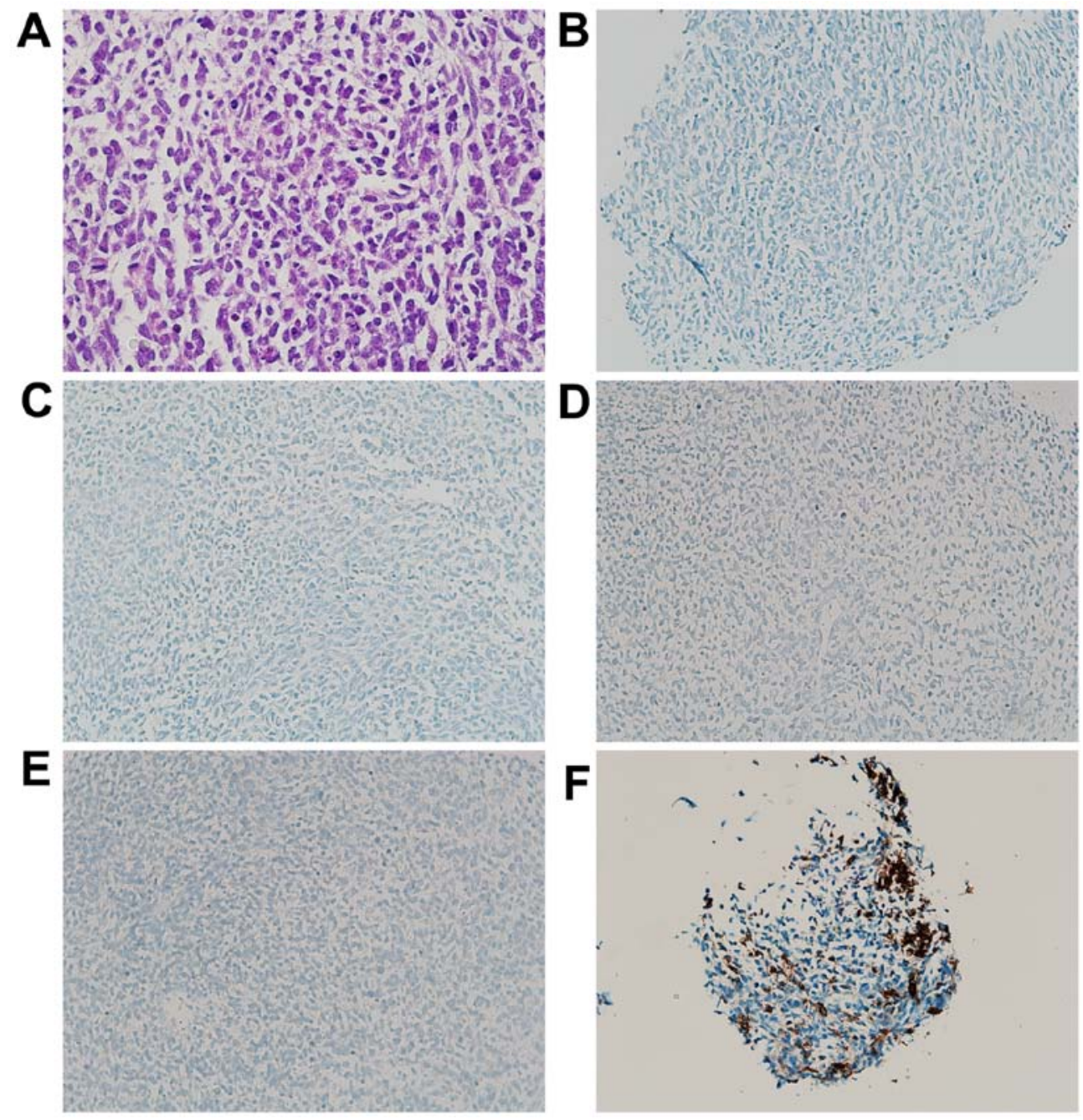

Figure 4. Photomicrographs of the left forearm tumor. (A) Diffuse proliferation of small round or polygonal cells with a high nuclear/cytoplasm ratio (H\&E stain; magnification, x200). The tumor was immunohistochemically negative for (B) S-100 (IHC stain; magnification, x100), (C) SOX10 (IHC stain; magnification, x100), (D) HMB45 (IHC stain; magnification, x100) and (E) Melan A (IHC stain; magnification, x100). The sample was also partially positive for (F) CD56 (IHC stain; magnification, x100). IHC, immunohistochemical; SOX10, SRY-related HMG-box 10; HMB45, human melanin black-45.

EWSR1-ATF1 fusion gene and changed its morphology to a GNET-like one. However, this theory has a limitation. The recurrent MM sample (Sample 2), which had been resected nearly seven years earlier, may have been too old for the detection of the EWSR1-ATF1 fusion gene. Fusion gene detection may have been falsely negative due to a sampling error.

Therefore, the combination of BRAF mutation analysis and EWSR1 fusion gene detection has attracted a significant amount of attention as a means of differentiating CCS from MM (13). Our patient's GNET exhibited both a BRAF mutation and EWSR1-ATF1 fusion genes, and combination therapy with dabrafenib mesylate and trametinib dimethyl sulfoxide proved to be temporarily effective. This combination of tests for GNET may contribute to the diagnosis as well as the choice of treatment method.

In summary, we reported the case of a woman with a history of desmoplastic MM exhibiting a BRAF mutation, which later transformed into a GNET exhibiting both a BRAF mutation and two subtypes of EWSR1-ATF1 fusion genes. Combination therapy with dabrafenib mesylate and trametinib dimethyl sulfoxide proved to be temporarily effective for this type of tumor. Further accumulation of similar cases will be necessary to elucidate the pathological significance of this type of tumor.

\section{Acknowledgements}

The authors would like to thank Dr Norifumi Naka (NachiKatsuura Town Onsen Hospital, Japan) for the supportive reading of this manuscript and useful suggestions.

\section{Funding}

No funding was received.

\section{Availability of data and materials}

The datasets used and/or analyzed during the present study are available from the corresponding author on reasonable request.

\section{Authors' contributions}

TYag conceived the present study. SN pathologically diagnosed the patient and revised the manuscript. TYam performed 
the gene analysis. TW, YI, HT, HS, KY and ST collected the clinical data. All authors read and approved the final manuscript prior to submission.

\section{Ethics approval and consent to participate}

The soft-tissue mass sample was collected after obtaining written informed consent from the patient according to a protocol approved by the Osaka International Cancer Institute (Osaka, Japan).

\section{Patient consent for publication}

Written informed consent was obtained from the patient's husband for the publication of her data and associated images.

\section{Competing interests}

The authors declare that they have no competing interests.

\section{References}

1. Stockman DL, Miettinen M, Suster S, Spagnolo D, DominguezMalagon H, Hornick JL, Adsay V, Chou PM, Amanuel B Vantuinen P and Zambrano EV: Malignant gastrointestinal neuroectodermal tumor: Clinicopathologic, immunohistochemical, ultrastructural, and molecular analysis of 16 cases with a reappraisal of clear cell sarcoma-like tumors of the gastrointestinal tract. Am J Surg Pathol 36: 857-868, 2012.

2. Chang B, Yu L, Guo WW, Sheng WQ, Wang L, Lao I, Huang D, Bai QM and Wang J: Malignant gastrointestinal neuroectodermal tumor: Clinicopathologic, immunohistochemical, and molecular analysis of 19 cases. Am J Surg Pathol 44: 456-466, 2020.

3. Green C, Spagnolo DV, Robbins PD, Fermoyle S and Wong DD: Clear cell sarcoma of the gastrointestinal tract and malignant gastrointestinal neuroectodermal tumor: Distinct or related entities? A review. Pathology 50: 490-498, 2018.

4. Antonescu CR, Tschernyavsky SJ, Woodruff JM, Jungbluth AA, Brennan MF and Ladanyi M: Molecular diagnosis of clear cell sarcoma: Detection of EWS-ATF1 and MITF-M transcripts and histopathological and ultrastructural analysis of 12 cases. J Mol Diagn 4: 44-52, 2002.
5. Enzinger FM: Clear-cell sarcoma of tendons and aponeuroses. An analysis of 21 cases. Cancer 18: 1163-1174, 1965.

6. Antonescu CR: Clear cell sarcoma of soft tissue. In: WHO Classification of Tumours of Soft tissue and Bone. 4th edition. Fletcher CDM, Bridge JA, Hogendoorn PCW and Mertens F (eds). International Agency for Research on Cancer (IARC), Lyon, pp221-222, 2013.

7. Zambrano E, Reyes-Mugica M, Franchi A and Rosai J: An osteoclast-rich tumor of the gastrointestinal tract with features resembling clear cell sarcoma of soft parts: Reports of 6 cases of a GIST simulator. Int J Surg Pathol 11: 75-81, 2003.

8. Thway $\mathrm{K}$ and Fisher C: Tumors with EWSR1-CREB1 and EWSR1-ATF1 fusions: The current status. Am J Surg Pathol 36: e1-e11, 2012.

9. Panagopoulos I, Mertens F, Dêbiec-Rychter M, Isaksson M, Limon J, Kardas I, Domanski H, Sciot R, Perek D, Crnalic S, et al: Molecular genetic characterization of the EWS/ATF1 fusion gene in clear cell sarcoma of tendons and aponeuroses. Int J Cancer 99: 560-567, 2002.

10. Wang WL, Mayordomo E, Zhang W, Hernandez VS, Tuvin D, Garcia L, Lev DC, Lazar AJF and López-Terrada D: Detection and characterization of EWSR1/ATF1 and EWSR1/CREB1 chimeric transcripts in clear cell sarcoma (melanoma of soft parts). Mod Pathol 22: 1201-1209, 2009.

11. Hocar O, Le Cesne A, Berissi S, Terrier P, Bonvalot S, Vanel D, Auperin A, Le Pechoux C, Bui B, Coindre JM and Robert C: Clear cell sarcoma (malignant melanoma) of soft parts: A clinicopathologic study of 52 cases. Dermatol Res Pract 2012: 984096, 2012.

12. Park BM, Jin SA, Choi YD, Shin SH, Jung ST, Lee JB, Lee SC and Yun SJ: Two cases of clear cell sarcoma with different clinical and genetic features: Cutaneous type with BRAF mutation and subcutaneous type with KIT mutation. Br J Dermatol 169: 1346-1352, 2013

13. Yang L, Chen Y, Cui T, Knösel T, Zhang Q, Geier C, Katenkam D and Petersen I: Identification of biomarkers to distinguish clear cell sarcoma from malignant melanoma. Hum Pathol 43: 1463-1470, 2012 International (CC BY-NC-ND 4.0) License. 